\title{
ThaNKS TO ReVIEWERS
}

The Editors gratefully acknowledge the generous assistance of the following individuals (apart from the Editorial Board) who reviewed manuscripts in 1999.

Vitaly Ablamunts

Steven J Ackerman

Ruben Adler

Simon Afford

Robert Aiello

Adriana Albini

Kari Alitalo

Christina Antonescu

Piero Anversa

Caroline B Appleyard

Suneel Aptee

Barry G Aranson

Wadih Arap

Douglas Arenberg

Sanford A Asher

Phillip Askenase

Hugh Auchincloss

Hellmut G Augustin

K Frank Austen

Marco Baggiolini

Allen Bale

Sanford H Barsky

Marc D Basson

Robert Baughman

Victoria Bautch

Pamela Becker

Richard Bell

Neal Benowitz

Michael Besich

Barbara Bierer

Holly Birdsall

Robert Bloch
Silvano Bosari

Alfred LM Bothwell

Michael Bowness

Janet L Brandsma

Douglas E Brash

Janice Brissette

Richard Bucala

Gerard Burrow

Joseph Califano

Guiseppina Caligiuri

James P Calvet

Sheldon Campbell

Gabriel Capella

Arnold Caplan

Bruce M Carlson

Graham Carpenter

Michael Carroll

Arturo Casadevall

Michael Centrella

Israel F Charo

Lucienne Chatenoud

Don W Cleveland

Lauren Cohn

David KC Cooper

Pierre A Coulombe

Joseph Craft

Carl E Creutz

Craig Crews

David Crowe

Christopher Crum

Bartalan Csillik

Ernest Cutz
Bogdan Czerniak

Agustin P Dalmasso

Keld Dano

Bernard Davis

Larissa Debelenko

David T Denhardt

Deborah Dillon

Daniel DiMaio

David E Dostal

Christopher Drake

Ann M Dvorak

Marie Egan

Oliver Eickelberg

Ellen Eisenberg

Peter Ekblom

Eva Engvall

William Evans

Mark Ewen

John L Farber

Eva Feldman

Susan J Fisher

Jonathan A Fletcher

Hussein Foda

Agnes Fogo

Werner W Franke

Matthew Frosch

Michiko Fukuda

Edward Gabrielson

Fred Gage

Warren Gallin

Pierluigi Gambetti

Frank N Gellerich 


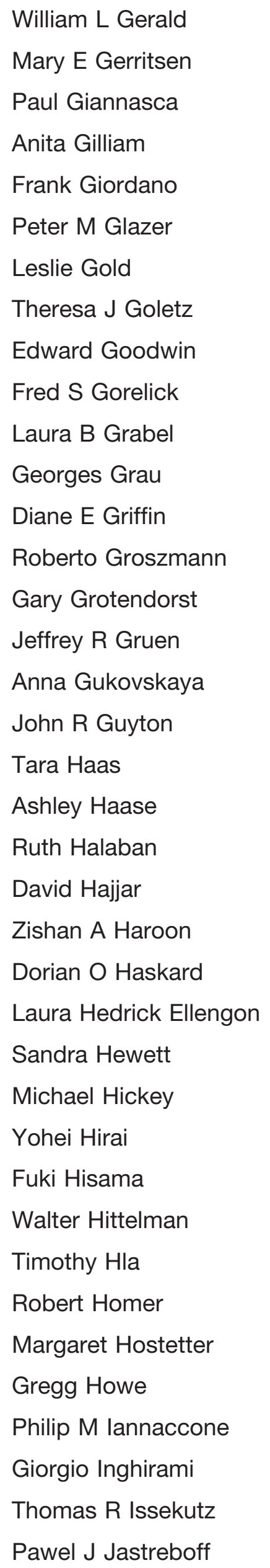

Jeffrey S Kahn

Linda Kalikin

Olli Kallioniemi

Raj Kapur

Saul Karpen

Clifford Kashtan

Dontscho Kerjaschki

Elliott Kieff

Bonnie King

Robert Kisilevsky

Warren Knudson

Hilary Koprowski

Michael Korn

Elizabeth J Kovacs

Paul H Krebsbach

Jordan Kreidberg

Paul Kubes

Laura Lamps

Lucia Languino

Yuri Lazebnik

Leslie Leinwand

Edward H Leiter

Thomas L Lentz

Robert M Levin

William Lewis

David N Liebowitz

W Marston Linehan

Daniel S Longnecker

Marc Lorber

Janet Lord

Thomas Maciag

Rizgar Mageed

Sergei Malkhosyan

Mark J Mamula

Timothy McCaffrey

Daniel Medina

Didier Merlin

Barbara Meyrick
George Miller

Gil Mor

John Spencer Mort

Hugo W Moser

David Muir

Janice Nagy

Victor Navarro

Peter J Neame

Leonard Neckers

Brian J Nickoloff

Santo V Nicosia

Yuri Nikiforov

Paul Noble

Asma Nusrat

Timothy J O'leary

Philip Osdoby

David L Page

Charles A Parkos

Archibald Perkins

George Perry

Manuel Perucho

Elizabeth Petty

Bruce R Pitt

Ellen Pizer

Nicholas Platt

Darwin Prockop

Vito Quaranta

Richard Quigg

Arnold B Rabson

Elaine Raines

Basil Rapoport

Michael A Reidy

Michael Reiss

Miguel Reyes-Mugica

David Rimm

Piero Rinaldo

Marie Robert

Allan Rofe 
Len Roza

Nancy H Ruddle

Jose Russo

Jeffrey Sample

Robert Sataloff

Angelo M Scanu

Jeffrey Schechner

Ann Marie Schmidt

Jan E Schnitzer

Stephen M Schwartz

Pauline Schwartz

Marvin L Sears

Margretta R Seashore

William Sessa

Leslie Shaw

Edward L Snyder

Michele Solimena

Paul Soloway

Lee Ann Sporn

Martin S Springer
Lawrence Steinman

Rose Stephens

David M Stern

William G Stetler-Stevenson

Freda Stevenson

R Strom

John P Sundberg

Carlos Taborda

Gerald Taborsky

Ivan Tack

Vernon L Tesh

Kenneth A Thomas

James G Tidball

Kevin Tracey

Alexandra Trkola

Jurg Tschopp

Eva Turley

Thomas R Ulich

Livingston Van De Water

James Varani
Arthur Veis

Agnes Vignery

David Viswanatha

D Wakefield

Fred Wang

Christian Weber

Scott Webster

Sherman Weissman

Rebecca Wells

Per Westermark

Ulla M Wewer

Kenneth R Williams

Susan Wong

Keping Xie

George Yancopoulos

Richard D Ye

Daniel Zelterman

Hui Zhang

Stanley Zucker 